$11-1-2012$

\title{
Posterior Estimates of Poisson Distribution Using R Software
}

\author{
Raja Sultan \\ University of Kashmir, Srinagar, $J$ \& $K$, India \\ S.P.Ahmad \\ University of Kashmir, Srinagar, J \& K, India
}

Follow this and additional works at: http://digitalcommons.wayne.edu/jmasm

Part of the Applied Statistics Commons, Social and Behavioral Sciences Commons, and the Statistical Theory Commons

\section{Recommended Citation}

Sultan, Raja and Ahmad, S.P. (2012) "Posterior Estimates of Poisson Distribution Using R Software," Journal of Modern Applied Statistical Methods: Vol. 11 : Iss. 2 , Article 24.

DOI: $10.22237 /$ jmasm/1351743780

Available at: http://digitalcommons.wayne.edu/jmasm/vol11/iss $2 / 24$

This Brief Report is brought to you for free and open access by the Open Access Journals at DigitalCommons@WayneState. It has been accepted for inclusion in Journal of Modern Applied Statistical Methods by an authorized editor of DigitalCommons@WayneState. 


\title{
Brief Report \\ Posterior Estimates of Poisson Distribution using R Software
}

\author{
Raja Sultan S. P. Ahmad \\ University of Kashmir, \\ Srinagar, J \& K, India
}

The Bayesian estimation of unknown parameter of the Poisson distribution is examined under different priors. The posterior distributions for the unknown parameter of the Poisson distribution are derived using the following priors: uniform, Jeffrey's, Gamma distribution, Gamma-Chi-square distribution, Gammaexponential distribution and Chi-square-exponential distribution. Numerical and graphical illustrations of the posterior densities of the parameters of interest were conducted using R Software.

Key words: Poisson distribution, prior distribution, posterior distribution, R software.

\section{Introduction}

The Poisson distribution was discovered in 1837 by French mathematician and physicist Simon Dennis Poisson (1781-1840). The Poisson distribution has many practical applications; one major area of application is in epidemiology, the study of disease incidence. The Poisson distribution arises naturally as a useful model for analyzing counts of rare events, such as the number of radioactive emissions in a fixed time interval or the number of bacteria in a given test sample.

The probability mass function (pmf) of the Poisson distribution of a random variable $Y$ having Parameter $\lambda$ is given by

$$
\begin{aligned}
& f(y)=\frac{e^{-\lambda} \lambda^{y}}{y !} \\
& y=0,1,2, \ldots \text { and } \lambda>0 .
\end{aligned}
$$

Raja Sultan is Research Scholar in the Department of Statistics. Email him at: hamzasultan18@yahoo.com. S. P. Ahmad is an Assistant Professor in the Department of Statistics. Email him at: sprvz@yahoo.com.
Haight's (1967) handbook provides many references regarding the Poisson distribution's applications in a variety of fields. Howlader and Balasooriya (2003), Bolstad (2007), Saleem and Aslam (2008), Tahir and Zawar (2008), Aslam and Haq (2009) and others have also discussed the distribution with applications.

Posterior Distribution of Unknown Parameter $\lambda$ Using Uniform Prior

If $y_{1}, y_{2}, \ldots, y_{n}$ are iid observations from an (1), then the likelihood function is

$$
L(\lambda)=\frac{e^{-n \lambda} \lambda^{\sum_{i=1}^{n} y_{i}}}{\prod_{i=1}^{n} y_{i}}
$$

Laplace (1774, 1812) found that it worked exceptionally well to always select the prior for $\lambda$ to be constant. Consider the uniform prior

$$
\begin{aligned}
& \mathrm{P}(\lambda) \alpha 1 \\
& 0<\lambda<\infty
\end{aligned}
$$

the posterior distribution $P(\lambda \mid Y)$ of the parameter $\lambda$ is found using (2) and (3) as 


$$
\begin{aligned}
& \mathrm{P}(\lambda \mid \mathrm{Y}) \alpha \mathrm{P}(\lambda) \\
& \Rightarrow \mathrm{P}(\lambda \mid \mathrm{Y})=\frac{\sum_{\mathrm{i}=1}^{\mathrm{n}} \mathrm{y}_{\mathrm{i}}+1}{\Gamma\left(\sum_{\mathrm{i}=1}^{\mathrm{n}} \mathrm{y}_{\mathrm{i}}+1\right)} \mathrm{e}^{-\mathrm{n} \lambda} \lambda^{\sum_{\mathrm{i}=1}^{\mathrm{n}} \mathrm{y}_{\mathrm{i}}},
\end{aligned}
$$

which is the density function of a gamma distribution with parameters $\left(n, \sum_{i=1}^{n} y_{i}+1\right)$.

Posterior Distribution of Unknown Parameter $\lambda$ Using Jeffrey's Prior

The Jeffrey's prior (see Berger, 1985) for the parameter $\lambda$ having distribution (1) is

$$
\mathrm{P}(\lambda) \quad \alpha \quad \lambda^{-\frac{1}{2}}
$$

and the posterior distribution $P(\lambda \mid Y)$ of the parameter $\lambda$ is found using (2) and (5) as

$$
\mathrm{P}(\lambda \mid \mathrm{Y})=\frac{\mathrm{n}^{\sum_{\mathrm{i}=1}^{\mathrm{n}} \mathrm{y}_{\mathrm{i}}+\frac{1}{2}}}{\Gamma\left(\sum_{\mathrm{i}=1}^{\mathrm{n}} \mathrm{y}_{\mathrm{i}}+\frac{1}{2}\right)} \mathrm{e}^{-\mathrm{n} \lambda} \lambda^{\sum_{\mathrm{i}=1}^{\mathrm{n}} \mathrm{y}_{\mathrm{i}}-\frac{1}{2}}
$$

which is the density function of a gamma distribution with parameters $\left(n, \sum_{i=1}^{n} y_{i}+\frac{1}{2}\right)$.

Posterior Distribution of Unknown Parameter $\lambda$ Using Gamma Distribution as Prior

The single prior distribution of $\lambda$ is a gamma distribution with hyper parameters a and $\mathrm{b}$ is

$$
\begin{aligned}
& \mathrm{P}(\lambda)=\frac{\mathrm{a}^{\mathrm{b}} \mathrm{e}^{-\mathrm{a} \lambda} \lambda^{\mathrm{b}-1}}{\Gamma(\mathrm{b})} \\
& \mathrm{a}>0, \mathrm{~b}>0, \lambda>0 .
\end{aligned}
$$

The posterior distribution $P(\lambda \mid Y)$ of parameter $\lambda$ is found using (2) and (7) as

$$
P(\lambda \mid Y)=\frac{(a+n){ }_{i=1}^{b+\sum_{i}^{n} y_{i}}}{\Gamma\left(b+\sum_{i=1}^{n} y_{i}\right)} e^{-\lambda(a+n)} \lambda^{\left(b+\sum_{i=1}^{n} y_{i}\right)-1},
$$

which is the density function of a gamma distribution with parameters $\left(a+n, b+\sum_{i=1}^{n} y_{i}\right)$.

Posterior Distribution of Unknown Parameter $\lambda$ Using Gamma-Chi-Square Prior

Assume that the prior distribution of $\lambda$ is a gamma distribution with hyper parameters $a_{1}$ and $b_{1}$ as

$$
\begin{aligned}
& \mathrm{P}_{1}(\lambda)=\frac{\mathrm{b}_{1}^{\mathrm{a}_{1}} \mathrm{e}^{-\lambda \mathrm{b}_{1}} \lambda^{\mathrm{a}_{1}-1}}{\Gamma\left(\mathrm{a}_{1}\right)} \\
& \mathrm{a}_{1}>0, \mathrm{~b}_{1}>0, \lambda>0
\end{aligned}
$$

The second prior assumed is a Chi-square distribution with hyper parameter $c_{1}$ given by

$$
\begin{aligned}
& \mathrm{P}_{2}(\lambda)=\frac{\left(\frac{1}{2}\right)^{\frac{\mathrm{c}_{1}}{2}} \mathrm{e}^{-\frac{\lambda}{2}} \lambda^{\frac{\mathrm{c}_{1}}{2}-1}}{\Gamma\left(\frac{\mathrm{c}_{1}}{2}\right)}, \\
& \mathrm{c}_{1}>0, \lambda>0 .
\end{aligned}
$$

A double prior is defined for $\lambda$ by combining the two priors in (9) and (10) as:

$$
\begin{aligned}
& \mathrm{P}(\lambda) \quad \alpha \quad \mathrm{P}_{1}(\lambda) \mathrm{P}_{2}(\lambda) \\
& \Rightarrow \mathrm{P}(\lambda) \propto \quad\left(\lambda^{\mathrm{a}_{1}-1} \mathrm{e}^{-\lambda\left(\mathrm{b}_{1}+\frac{1}{2}\right)} \lambda^{\frac{\mathrm{c}_{1}}{2}-1}\right) .
\end{aligned}
$$

The posterior distribution $P(\lambda \mid Y)$ of parameter $\lambda$ is found by using (2) and (11) as 


$$
\begin{aligned}
& P(\lambda \mid Y)= \\
& \frac{\left(n+b_{1}+\frac{1}{2}\right)^{a_{1}+\frac{c_{1}}{2}+\sum_{i=1}^{n} y_{i}-1}}{\Gamma\left(a_{1}+\frac{c_{1}}{2}+\sum_{i=1}^{n} y_{i}-1\right)} e^{-\lambda\left(n+b_{1}+\frac{1}{2}\right)} \lambda^{\left(a_{1}+\frac{c_{1}}{2}+\sum_{i=1}^{n} y_{i}-1\right)-1},
\end{aligned}
$$

which is the density function of gamma distribution with parameters

$$
\left(n+b_{1}+\frac{1}{2}, a_{1}+\frac{c_{1}}{2}+\sum_{i=1}^{n} y_{i}-1\right) \text {. }
$$

Posterior Distribution of Unknown Parameter $\lambda$ Using Gamma-Exponential Prior

The double prior for $\lambda$ is defined to be a gamma distribution with hyper parameters $\left(\mathrm{a}_{2}\right.$, $\mathrm{b}_{2}$ ) and an exponential distribution with hyper parameter $\mathrm{c}_{2}$ as

$$
\begin{aligned}
& \mathrm{P}(\lambda) \alpha \quad \lambda^{\mathrm{a}_{2}-1} \mathrm{e}^{-\lambda\left(\mathrm{b}_{2}+\mathrm{c}_{2}\right)}, \\
& \lambda>0 .
\end{aligned}
$$

The posterior distribution $P(\lambda \mid Y)$ of parameter $\lambda$ is given by using (2) and (12) as

$$
\begin{aligned}
& P(\lambda \mid Y)= \\
& \frac{\left(n+b_{2}+c_{2}\right)^{a_{2}+\sum_{i=1}^{n} y_{i}}}{\Gamma\left(a_{2}+\sum_{i=1}^{n} y_{i}\right)} e^{-\lambda\left(n+b_{2}+c_{2}\right)} \lambda^{\left(a_{2}+\sum_{i=1}^{n} y_{i}\right)-1},
\end{aligned}
$$

which is the density function of a gamma distribution with parameters $\left(n+b_{2}+c_{2}, a_{2}+\sum_{i=1}^{n} y_{i}\right)$.

Posterior Distribution of Unknown Parameter $\lambda$ Using Chi-Square and Exponential Prior

The double prior for $\lambda$ is defined to be a Chi-square distribution with hyper parameter $a_{3}$ and an exponential distribution with hyper parameter $\mathrm{c}_{3}$ as

$$
\begin{aligned}
& \mathrm{P}(\lambda) \alpha \lambda^{\frac{\mathrm{a}_{3}}{2}-1} \mathrm{e}^{-\lambda\left(\mathrm{c}_{3}+\frac{1}{2}\right)}, \\
& \lambda>0 .
\end{aligned}
$$

The posterior distribution $P(\lambda \mid Y)$ of parameter $\lambda$ is found by using (2) and (13) as

$$
\begin{aligned}
& \mathrm{P}(\lambda \mid \mathrm{Y})= \\
& \frac{\left(\mathrm{n}+\mathrm{c}_{3}+\frac{1}{2}\right)^{\frac{\mathrm{a}_{3}}{2}+\sum_{\mathrm{i}=1}^{\mathrm{n}} \mathrm{y}_{\mathrm{i}}}}{\Gamma\left(\frac{\mathrm{a}_{3}}{2}+\sum_{\mathrm{i}=1}^{\mathrm{n}} \mathrm{y}_{\mathrm{i}}\right)} \mathrm{e}^{-\lambda\left(\mathrm{n}+\mathrm{c}_{3}+\frac{1}{2}\right)} \lambda^{\left(\frac{\mathrm{a}_{3}}{2}+\sum_{\mathrm{i}=1}^{\mathrm{n}} \mathrm{y}_{\mathrm{i}}\right)-1},
\end{aligned}
$$

which is the density function of gamma distribution with parameters $\left(n+c_{3}+\frac{1}{2}, \frac{a_{3}}{2}+\sum_{i=1}^{n} y_{i}\right)$.

\section{Numerical Illustration}

\section{Methodology}

The Poisson distribution provides a realistic model for many random phenomena. Because the values of a Poisson random variable are non-negative integers, any random phenomena for which a count is of interest is a candidate for modeling by assuming a Poisson distribution. The numerical and graphical illustration of posterior densities of the parameters of interest conveys a convincing and comprehensive picture of Bayesian data analysis.

Several programs were developed to calculate posterior densities of the Poisson distribution under various priors in R Software for this study. These programs illustrate the strength of Bayesian methods in practical situations. Data analyzed for this study is from Hoff (2009): During the 1990s the General Social Survey gathered data on educational attainment and number of children among 155 women who were 40 years of age at the time of their participation in the survey. Let $Y_{1,1}, Y_{2,1}, \ldots, Y_{n 1,1}$ denote the number of children for $\mathrm{n}_{1}$ women without college degrees and $Y_{1,2}, Y_{2,2}, \ldots, Y_{n 2,2}$ denote the number of children 
for $\mathrm{n}_{2}$ women with college degrees. The group sums and means are:

Group I, Less than bachelors:

$$
\begin{gathered}
\mathrm{n}_{1}=111, \\
\sum_{\mathrm{i}=1}^{\mathrm{n}_{1}} \mathrm{Y}_{\mathrm{i}, 1}=217, \\
\bar{Y}_{1}=1.95
\end{gathered}
$$

Group II, Bachelors or higher:

$$
\begin{gathered}
\mathrm{n}_{2}=44, \\
\sum_{\mathrm{i}=1}^{\mathrm{n}_{2}} \mathrm{Y}_{\mathrm{i}, 2}=66, \\
\bar{Y}_{1}=1.50
\end{gathered}
$$

the posterior mean and posterior variance of parameter $\lambda$ are presented in Table 1 for Groups I and II under different types of priors. Graphical displays of posterior densities for $\lambda$ under different priors are shown in Figures 1 and 2.

Figure 1: Posterior Distribution of Mean Birth Rate for Group I

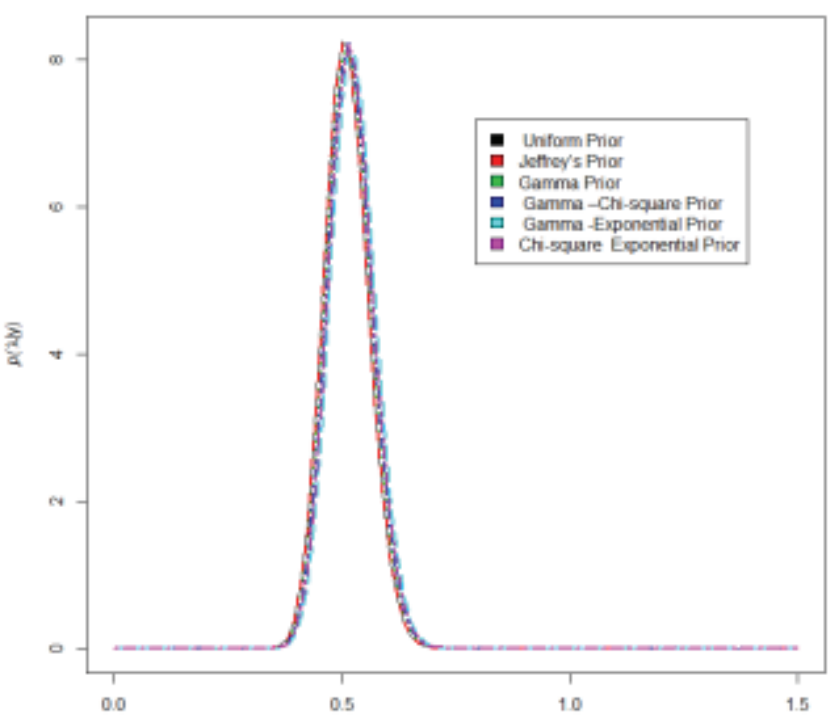

Figure 2: Posterior Distribution of Mean Birth Rate for Group II

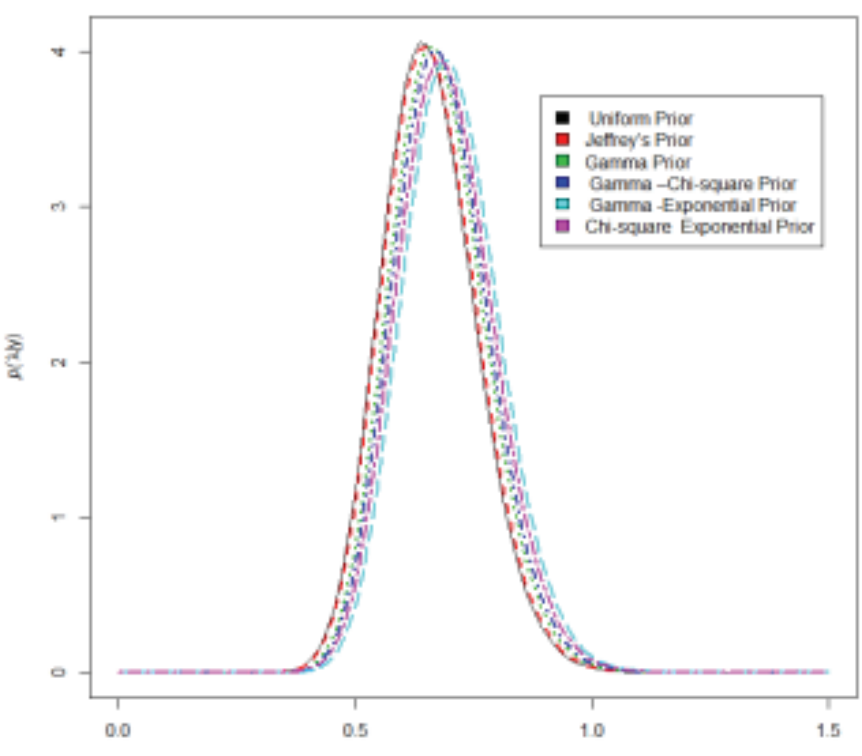

Results

Table 2 shows that the posterior mean for Group I (less than bachelors) under all assumed priors is higher than that of Group II (bachelors or higher). The posterior variance for Group I (less than bachelors) is less than the posterior variance of Group II (bachelors or higher) under all assumed priors. The posterior variance under all the assumed priors is calculated assuming the values of all hyper parameters are 2. Table 2 also shows that the posterior variance under the double prior Gamma-Exponential distribution is less compared to other assumed priors; thus, this prior is more efficient compared to other priors and the lower variation in posterior distribution assists in more precise Bayesian estimates of the true unknown parameter $\lambda$ of a Poisson distribution.

\section{References}

Aslam, M., \& Haq, A. (2009). On the prior selection for variance of normal distribution. Interstat, \#005.

Berger, J. O. (1985). Statistical decision theory and Bayesian analysis, $2^{\text {nd }}$ Edition. New York, NY: Springer-Verlag. 
POSTERIOR ESTIMATES OF POISSON DISTRIBUTION USING R SOFTWARE

Table 1: Posterior Mean and Posterior Variance of Parameter $\lambda$ of a Poisson Distribution with Different Priors

\begin{tabular}{|c|c|c|c|}
\hline Type of Prior & Prior Distribution & Posterior Mean & Posterior Variance \\
\hline Uniform Prior & 1 & $\frac{\sum_{i=1}^{n} y_{i}+1}{n}$ & $\frac{\sum_{i=1}^{n} y_{i}+1}{n^{2}}$ \\
\hline Jeffrey's Prior & $\lambda^{-\frac{1}{2}}$ & $\frac{\sum_{i=1}^{n} y_{i}+\frac{1}{2}}{n}$ & $\frac{\sum_{i=1}^{n} y_{i}+\frac{1}{2}}{n^{2}}$ \\
\hline Gamma Distribution & $e^{-a \lambda} \lambda^{b-1}$ & $\frac{b+\sum_{i=1}^{n} y_{i}}{a+n}$ & $\frac{b+\sum_{i=1}^{n} y_{i}}{(a+n)^{2}}$ \\
\hline Gamma-Chi-Square Distribution & $\lambda^{a_{1}-1} e^{-\lambda\left(b_{1}+\frac{1}{2}\right)} \lambda^{\frac{c_{1}}{2}-1}$ & $\frac{a_{1}+\frac{c_{1}}{2}+\sum_{i=1}^{n} y_{i}-1}{n+b_{1}+\frac{1}{2}}$ & $\frac{a_{1}+\frac{c_{1}}{2}+\sum_{i=1}^{n} y_{i}-1}{\left(n+b_{1}+\frac{1}{2}\right)^{2}}$ \\
\hline Gamma-Exponential Distribution & $\lambda^{a_{2}-1} e^{-\lambda\left(b_{2}+c_{2}\right)}$ & $\frac{a_{2}+\sum_{i=1}^{n} y_{i}}{n+b_{2}+c_{2}}$ & $\frac{a_{2}+\sum_{i=1}^{n} y_{i}}{\left(n+b_{2}+c_{2}\right)^{2}}$ \\
\hline Chi-Square-Exponential Distribution & $\lambda^{\frac{a_{3}}{2}-1} e^{-\lambda\left(c_{3}+\frac{1}{2}\right)}$ & $\frac{\frac{a_{3}}{2}+\sum_{i=1}^{n} y_{i}}{n+c_{3}+\frac{1}{2}}$ & $\frac{\frac{a_{3}}{2}+\sum_{i=1}^{n} y_{i}}{\left(n+c_{3}+\frac{1}{2}\right)^{2}}$ \\
\hline
\end{tabular}


SULTAN\& AHMAD

Table 2: Posterior Mean and Posterior Variance of a Poisson Distribution with Different Priors

\begin{tabular}{|c|c|c|c|c|}
\hline \multirow{2}{*}{ Type Of Prior } & \multicolumn{2}{|c|}{ Less Than Bachelor's } & \multicolumn{2}{c|}{ Bachelors Or Higher } \\
\cline { 2 - 5 } & Posterior Mean & $\begin{array}{c}\text { Posterior } \\
\text { Variance }\end{array}$ & Posterior Mean & $\begin{array}{c}\text { Posterior } \\
\text { Variance }\end{array}$ \\
\hline Uniform Prior & 1.963964 & 0.01769337 & 1.522727 & 0.03460744 \\
\hline Jeffrey's Prior & 1.959459 & 0.01765279 & 1.511364 & 0.03434917 \\
\hline $\begin{array}{c}\text { Gamma Distribution } \\
\text { Gamma-Chi-Square } \\
\text { Distribution }\end{array}$ & 1.938053 & 0.01715091 & 1.478261 & 0.03213611 \\
\hline $\begin{array}{c}\text { Gamma-Exponential } \\
\text { Distribution }\end{array}$ & 1.904348 & 0.01655955 & 1.416667 & 0.02951389 \\
\hline $\begin{array}{c}\text { Chi-Square-Exponential } \\
\text { Distribution }\end{array}$ & 1.920705 & 0.01692251 & 1.440860 & 0.03098624 \\
\hline
\end{tabular}

Bolstad, W. M. (2007). Introduction to Bayesian statistics. New York, NY: John Wiley $\&$ Sons.

Haight, F. A. (1967). Handbook of the Poisson distribution. New York, NY: John Wiley \& Sons.

Hoff, P. D. (2009). A first course in Bayesian statistical methods. New York, NY: Springer.

Howlader, H. A., \& Balasooriya, U. (2003). Bayesian estimation of the distribution function of the Poisson model. Biometrical Journal, 45(7), 901-912.
Laplace, P. S. (1774, trans 1986). Memoire sur la probabilite des causes par les evenemens. Translation by S. Stigler. Memoir on the probability of causes of events. Statistical Science, 1, 364-378.

Laplace, P. (1812). Theorie Analytique des Probabilite's. Paris: Courcier.

Saleem, M., \& Aslam, M. (2008). On the prior selection for the mixture of Rayleigh distribution using predictive intervals. Pakistan Journal of Statistics, 24(1), 21-35.

Tahir, M., \& Zawar, H. (2008).Comparison of non-informative priors for number of defects (Poisson) model. Interstat, \#002. 